\title{
Some factors controlling the interaction between response-dependent and response-independent schedules of reinforcement
}

\author{
JACK E. SHERMAN \\ University of Colorado, Boulder, Colorado 80302 \\ and \\ JOSEPH H. SPITZNER \\ Ohio State University, Columbus 43210
}

\begin{abstract}
Response rate interactions were studied in four rats following a shift from: (a) a multiple schedule in which two stimuli, $S_{1}$ and $S_{2}$, were both correlated with response-dependent reinforcement (mult VI $1 \mathrm{~min}$ VI $1 \mathrm{~min}$ ) to (b) a multiple schedule in which $\mathrm{S}_{2}$ was now correlated with response-independent reinforcement (mult VI 1 min VT $1 \mathrm{~min}$ ). Concomitant with the decrease in the rate of responding in the VT component, there was also a decrease in rate of responding during the VI component, i.e., negative induction. The nature of the observed interaction suggested that responding appropriate to the VT component may have generalized to the VI component. To test this interpretation, in a second experiment an effort was made to accentuate the differences between the components of the multiple schedules and consequently to reduce the degree of interaction. For four rats, two had a different reinforcer correlated with each component and two had a different response lever correlated with each component. The results of the separate-reinforcer treatment were not consistently different from those results obtained in Experiment I. The separate-lever treatment, however, resulted in decreased negative induction interaction and lower rates of responding during the VT component.
\end{abstract}

With multiple schedules in which one stimulus is correlated with a variable interval (responsedependent) schedule of reinforcement and is successively alternated with a second stimulus correlated with a comparable variable-time (responseindependent) schedule of reinforcement, it has been demonstrated with pigeons and rats that the VI component will control higher rates of responding (Halliday \& Boakes, 1971; Huff, Sherman, \& Cohn, 1975; Lattal \& Maxey, 1971; Weisman \& Ramsden, 1973; Wilkie, 1972). Among those studies which have observed baseline performance on a multiple variable-interval variable-interval schedule (mult VI VI) and then shifted to a multiple variable-interval variable-time schedule (mult VI VT) two unique interactions can be characterized. These are: (a) an enhanced maintenance of responding during the VT component relative to the rate maintained after shifting from a simple VI to a simple VT and (b) a decrease in response rate during VI concomitant with the decrease during VT.

The first experiment here was a systematic replication of previous studies shifting from a mult VI

This study was conducted as an $\mathbf{M}$. A. thesis at The Ohio State University by the first author under the direction of the second author. This paper is sponsored by David R. Thomas. who takes full editorial responsibility for its contents. Reprint requests should be addressed to Jack E. Sherman. Department of Psychology. University of Colorado. Boulder, Colorado 80302.
VI to a mult VI VT. The obtained results were consistent with those of previous studies: notably, the above-mentioned interactions were observed. The second experiment attempted to isolate some of the factors mediating these interactions.

\section{EXPERIMENT I}

\section{Method}

Subjects. Four naive albino male rats were maintained at $80 \%$ of their free-feeding adult weights throughout the experiment. The rats were Sprague-Dawley strain descendants obtained from the Laboratory Supply Company. Indianapolis, Indiana.

Apparatus. Two standard operant conditioning chambers (Foringer), each equipped with a single lever requiring a minimum of $.20 \mathrm{~N}$ to operate the microswitch were employed. The delivery of Noyes $.045 \mathrm{gg}$ food pellets served as reinforcers. Ventilation fans and white noise masked extraneous sound. The programmed stimuli were either a $3 / \mathrm{sec}$ click delivered through a $3.81-\mathrm{cm}$ speaker house in the rear of the chamber, or the absence of that click. Standard automated programming and recording equipment were located in an adjacent room.

Procedure. On the 1st day of training. all rats were habituated to the chamber and trained to approach the pellet tray when the food magazine click signaled the availability of a pellet. On the 2 nd day, all rats were shaped to press the lever and received continuous reinforcement for a total of 100 pellets for that day. On Day 3. each rat received 10 pellets on a continuous reinforcement schedule and then was shifted to a VI 1-min schedule for a total of 100 reinforcements. The VI schedule was generated by an arithmetic series of randomly arranged intervals varying from 7.8 to $120 \mathrm{sec}$.

On Day 4, each rat was exposed to a mult VI 1-min VI 1-min schedule of reinforcement. The component schedules alternated every 2.4 , or $6 \mathrm{~min}$ in a random order. After rate of responding had 


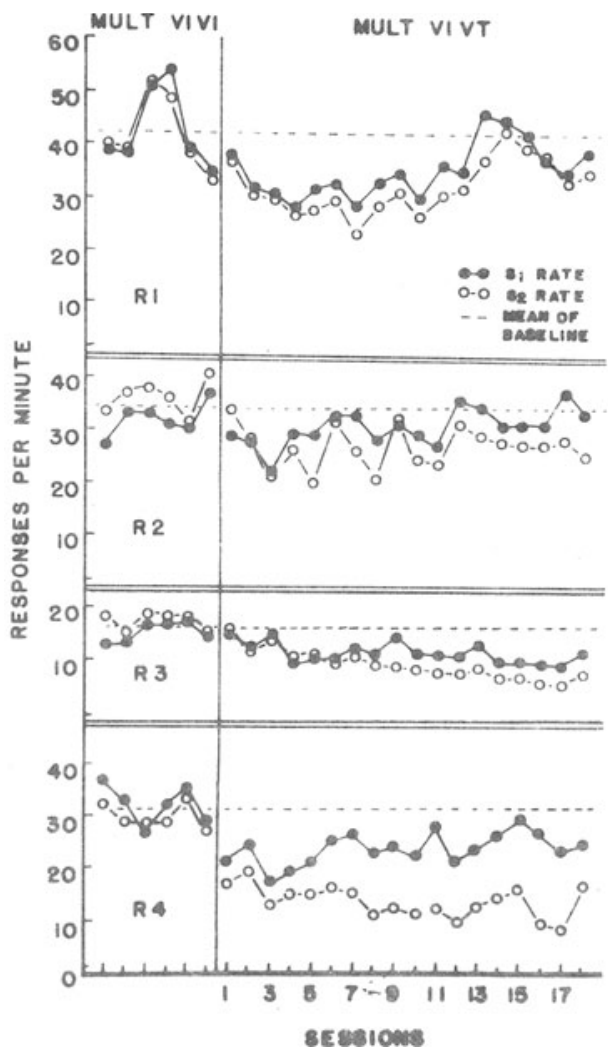

Figure 1. Rates of responding in each component of the multiple schedules for Experiment 1. $S_{1}$ [VI] refers to the clickon stimulus for Rats 1 and 4 and to the click-off stimulus for Rats

stabilized on mult VI VI, the component schedule correlated with one of the stimuli was shifted from VI to VT (mult VI VT). The criterion of stability used was a ratio of the mean response rate of the first three sessions divided by the mean of the last three sessions of six successive sessions $\geqslant .95$. The VT schedule was generated by the same interval tape used for the VI schedule. For subjects R-2 and R-3, click-on was correlated with the VT component, and for R-1 and R-4 click-off was correlated with the VT component. All rats were exposed to mult VI VT for 18 sessions. The rats were run $2 \mathrm{~h} /$ day, $60 \mathrm{~min}$ in each component, 7 days a week.

\section{Results}

Rates stabilized on the mult VI VI by Sessions 24, 24, 20, and 36 for Subjects R-1, R-2, R-3, and R-4, respectively (see Figure 1). Following the shift from mult VI VI to mult VI VT, there was a decrease in the rate of responding during the stimulus correlated with the VT component for all subjects within three sessions. Concomitant with the decrease in rate during the VT component, there was also a decrease in rate during the VI component. This class of interaction has been termed negative induction (Reynolds, 1961). The rate of responding in the VI component decreased (negative) and changed direction to that prevailing in the VT component.

A ratio was computed to summarize the changes in the rate of responding during each component of the mult VI VT relative to the stable rate maintained during each component of the last six sessions of mult
VI VI. This rate-change ratio was calculated for each component over three successive blocks of six mult VI VT (Sessions 1-6, 7-12, and 13-18). The ratio was computed from the formula: the average rate of responding over six sessions in component $\mathrm{X}$ during mult VI VT divided by the average rate of responding over the last six sessions in Component $\mathrm{X}$ of mult VI VI, where $X$ is the component correlated with the same stimulus in both mult VI VI and mult VI VT for each rat. A ratio value equal to 1.00 indicates no change in the rate of responding from baseline and an increase is indicated by a value $>1.00$, a decrease by a value $<1.00$.

Tabie 1 presents this summary information for each rat. The negative induction effect is reflceted in Table 1 by the concomitant decrease of the ratio values in both the VI and VT components. For R-1, $\mathrm{R}-2$, and $\mathrm{R}-4$, there is a gradual recovery of rate responding during the VI component as reflected by the increasing ratio values. R-3 does not show this gradual increase in rate during the VI component but a continued decrease.

The discrimination ratios presented in Table 1 indicate the proportion of responding in the VI component relative to the total number of responses over blocks of six sessions during mult VI VI and mult VI VT. Ratio values $>.50$ indicate that higher rates were obtained in the VI component, while ratio values $<.50$ indicate that higher rates were obtained during the VT component. For all subjects, excluding R-1, an increase in the relative proportion of responses in the VI component was observed over sessions.

\section{Discussion}

The negative induction effect reported here was more sustained and pronounced than has been observed in other studies with rats following a similar schedule shift from mult VI VI to mult VI VT (Lattal \& Maxey, 1971; Wilkie, 1972). It is not clear

Table 1

Response-Change Ratio and Discrimination Ratio for Experiment I

\begin{tabular}{|c|c|c|c|c|c|c|}
\hline \multirow[b]{2}{*}{ Subjects } & \multirow[b]{2}{*}{ Ratio } & \multirow{2}{*}{$\frac{\begin{array}{c}\text { Sessions } \\
\text { Mult VI VI }\end{array}}{\text { Last } 6}$} & \multicolumn{4}{|c|}{$\begin{array}{c}\text { Sessions } \\
\text { Mult VI VT }\end{array}$} \\
\hline & & & & $1-6$ & $7-12$ & $13-18$ \\
\hline \multirow{3}{*}{$\mathrm{R}-1$} & $\mathrm{RC}^{*}$ & VI & VI & .76 & .78 & .96 \\
\hline & $\mathrm{RC}$ & VI & VT & .72 & .68 & .85 \\
\hline & $\mathrm{DR} \dagger$ & .50 & & .52 & .54 & .53 \\
\hline \multirow{3}{*}{$\mathrm{R}-2$} & $\mathrm{RC}$ & VI & VI & .89 & .96 & 1.03 \\
\hline & $\mathrm{RC}$ & VI & VT & .75 & .72 & .71 \\
\hline & DR & .51 & & .51 & .54 & .56 \\
\hline \multirow{3}{*}{$\mathrm{R}-3$} & $\mathrm{RC}$ & VI & VI & .78 & .77 & .68 \\
\hline & $\mathrm{RC}$ & VI & VT & .68 & .59 & .49 \\
\hline & DR & .47 & & .50 & .54 & .55 \\
\hline \multirow{3}{*}{$\mathrm{R}-4$} & $\mathrm{RC}$ & VI & VI & .66 & .70 & .78 \\
\hline & $\mathrm{RC}$ & VI & VT & .54 & .41 & .44 \\
\hline & DR & .52 & & .57 & .65 & .66 \\
\hline
\end{tabular}

Note-See text for explanation.

${ }^{*} R C=$ rate-change ratio.

$\dagger D R=$ discrimination ratio. 
what aspect of the present procedure may have produced this enhanced interaction since the present procedure differed from those employed in these other studies with respect to stimuli, density of reinforcement, component alternation, and session length.

The maintenance of high rates of responding during the VT component of the mult VI VT confirmed an observation by Lattal and Maxey (1971). These authors stated that, compared to the rate maintained after shifting from a simple VI to a simple VT schedule (Rescorla \& Skucy, 1969), rate maintained in the VT component of the mult VI VT was much higher.

Based on the nature of the interactions observed, it is plausible that following the introduction of mult VI VT, responding appropriate to each component considered separately generalizes to the other component when in the context of a multiple schedule. More specifically, responding during the VT component of a mult VI VT may be maintained at a higher rate than on a simple VT schedule alone because operant responding maintained on the VI component may occur or generalize to the VT component. Conversely, the stable rates maintained on the VI component during baseline may decrease on the VI component of the mult VI VT schedule as a function of an increase in competing behaviors reinforced adventitiously on the VT component (Davis \& Hubbard, 1972) occurring during the VI component. At a more cognitive level of interpretation, the decreased rate on the VI component may be a function of a generalized 'laziness' resulting from free food delivery during the VT component (Engberg, Hansen, Welker, \& Thomas, 1972).

\section{EXPERIMENT II}

If generalization is part of the process mediating the observed interactions in Experiment I, then accentuating the differences between the components should decrease the magnitude of the observed interactions. In the first treatment, the differences between the components of the multiple schedules were accentuated by correlating a different reinforcer with each component. The reinforcer was either a dry pellet or limited access to a sucrose solution. In the second treatment, a different operandum was correlated with each component of the multiple schedule. This was accomplished by alternating the availability of two levers; each lever was correlated with a single component.

\section{Method}

Subjects. Four naive male rats of the same strain as in Experiment I were maintained at $80 \%$ of their free-feeding adult weights throughout the experiment.

Apparatus and procedure. The same apparatus and procedure were used in Experiment II as in Experiment I except as indicated below.

Separate reinforcer treatment. Subjects SR-1 and SR-2 were run in one of the same experimental chambers used in Experiment $I$. This chamber was modified with the addition of a dipper mechanism. The dipper delivered a $16 \%$ sucrose solution $(.2 \mathrm{cc})$ for $2 \mathrm{sec}$ through a rectangular opening centered on the front wall to the side of the food-pellet tray. SR-1 was first shaped to respond on the lever with sucrose and then presented food pellets. SR-2 received the reversed order. The shaping procedure was identical to that described in Experiment I except that the first half of the reinforcers delivered were either sucrose or food pellets.

Beginning with the first experimental session (Day 4), the sucrose reinforcer was paired with the click-off stimulus, and the pellet

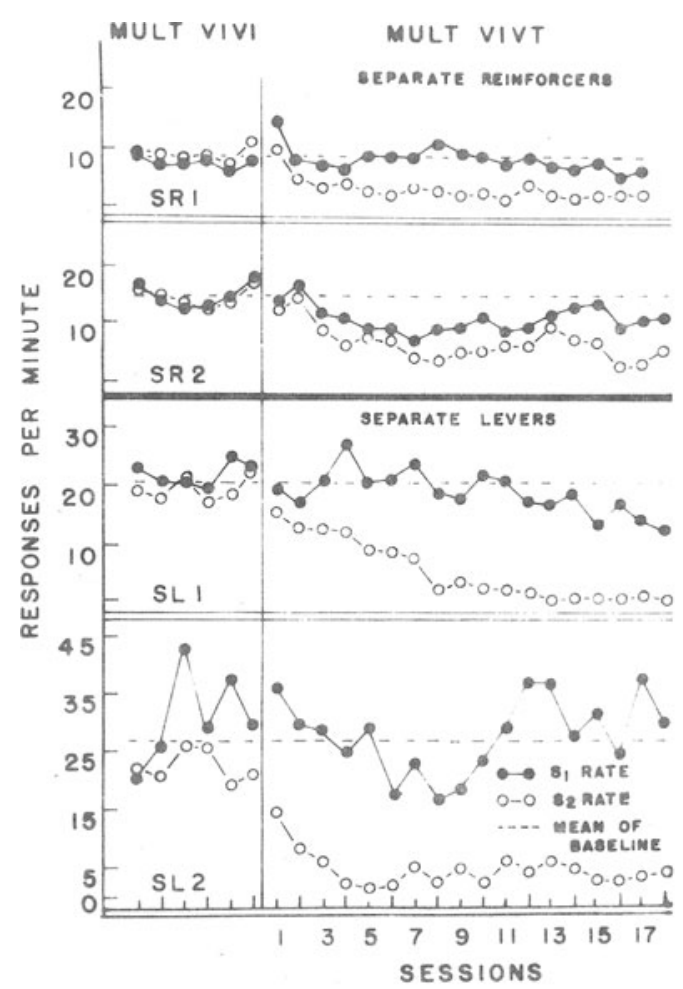

Figure 2. Rates of responding in each component of the multiple schedules for Experiment $\mathrm{II}_{\text {. }} \mathrm{S}_{1}[\mathrm{VI}]$ refers to sucrose and click-off condition for SR-1 and to pellet and click-on for SR-2. For the separate lever treatment, $S_{1}[V I]$ refers to the left lever for SL-1 and to the right lever for SL-2. Note that SR-1 inadvertently received only 17 sessions of mult VI VT.

reinforcer was paired with the click-on stimulus for both subjects. After responding stabilized on the mult VI VI schedule, one component of the multiple schedule was shifted to a VT schedule. For SR-1 click-on (pellet) was correlated with the VT component, and for SR-2 click-off (sucrose) was correlated with the VT component.

Separate lever treatment. Subjects SL-1 and SL-2 were trained in a Plexiglas chamber equipped with two retractable levers which alternated in their availability. The two levers were laterally located, $3 \mathrm{~cm}$ to either side of the food pellet tray. A minimum of approximately $.30 \mathrm{~N}$ was required to activate the microswitch of either lever. SL-1 was initially shaped to press the left lever and then the right lever with food pellets serving as the reinforcer while SL-2 received the reversed order. The alternating levers provided the only programmed discriminative cues during the multiple schedules. Each lever was correlated with a single component of the multiple schedule. When rate stabilized on mult VI VI, one of the levers was then shifted to a VT schedule. For SL-1, the right lever was correlated with the VT component while for SL-2 the left lever was correlated with the VT component.

\section{Results}

Separate reinforcer treatment. Response rate stabilized on the mult/VI for Sr-1 and SR-2 by Sessions 21 and 9, respectively. Rate of responding was higher during the VI component correlated with the click on stimulus (pellet) for both subjects during mult VI VI (see Figure 2). 
Table 2

Response-Change Ratio and Discrimination Ratio for Experiment II

\begin{tabular}{|c|c|c|c|c|c|c|}
\hline \multirow[b]{2}{*}{ Subjects } & \multirow[b]{2}{*}{ Ratio } & \multirow{2}{*}{$\begin{array}{c}\begin{array}{c}\text { Sessions } \\
\text { Mult VI VI }\end{array} \\
\text { Last } 6\end{array}$} & \multicolumn{4}{|c|}{$\begin{array}{c}\text { Sessions } \\
\text { Mult VI VT } \\
\end{array}$} \\
\hline & & & & $1-6$ & $7-12$ & $13-18$ \\
\hline SR-1 & $\begin{array}{l}\mathrm{RC}^{*} \\
\mathrm{RC} \\
\mathrm{DR} \dagger\end{array}$ & $\begin{array}{l}\text { VI } \\
\text { VI } \\
\quad .46\end{array}$ & $\begin{array}{l}\text { VI } \\
\text { VT }\end{array}$ & $\begin{array}{r}1.16 \\
.53 \\
.65\end{array}$ & $\begin{array}{r}1.19 \\
.32 \\
.76\end{array}$ & $\begin{array}{l}.86 \\
.22 \\
.77\end{array}$ \\
\hline SR-2 & $\begin{array}{l}\mathrm{RC} \\
\mathrm{RC} \\
\mathrm{DR}\end{array}$ & $\begin{array}{l}\text { VI } \\
\text { VI } \\
\quad .43\end{array}$ & $\begin{array}{l}\text { VI } \\
\text { VT }\end{array}$ & $\begin{array}{l}.82 \\
.63 \\
.56\end{array}$ & $\begin{array}{l}.61 \\
.35 \\
.63\end{array}$ & $\begin{array}{l}.79 \\
.40 \\
.66\end{array}$ \\
\hline SL-1 & $\begin{array}{l}\text { RC } \\
\text { RC } \\
\text { DR }\end{array}$ & $\begin{array}{l}\text { VI } \\
\text { VI } \\
\quad .53\end{array}$ & $\begin{array}{l}\text { VI } \\
\text { VT }\end{array}$ & $\begin{array}{l}.95 \\
.61 \\
.64\end{array}$ & $\begin{array}{l}.90 \\
.16 \\
.86\end{array}$ & $\begin{array}{l}.69 \\
.03 \\
.96\end{array}$ \\
\hline SL-2 & $\begin{array}{l}\text { RC } \\
\text { RC } \\
\text { DR }\end{array}$ & $\begin{array}{l}\text { VI } \\
\text { VI } \\
\quad .57\end{array}$ & $\begin{array}{l}\text { VI } \\
\text { VT }\end{array}$ & $\begin{array}{l}.90 \\
.25 \\
.83\end{array}$ & $\begin{array}{l}.78 \\
.18 \\
.86\end{array}$ & $\begin{array}{r}1.01 \\
.17 \\
.89\end{array}$ \\
\hline
\end{tabular}

Note-See text for explanation.

${ }^{*} R C=$ rate-change ratio.

$\dagger D R=$ discrimination ratio.

Following the introduction of mult VI VT, the rate of responding during VT gradually decreased over the 18 mult VI VT sessions for both subjects. A negative induction effect was observed for SR-2 but not for SR-1 . Both subjects demonstrated a greater decrease in the rate-change ratio (described in Experiment I) in VT than did the subjects in Experiment I (see Table 2). The discrimination ratio showed the same general increases as with all other subjects in Experiment I.

Separate lever treatment. Response rate stabilized on mult VI VI for SL-1 by Session 22. SL-2 failed to meet the stability criterion as defined in Experiment I and was shifted to mult VI VT following Session 26 on mult VI VI. For both subjects, a higher rate was maintained during mult VI VT on the lever they were initially shaped to press (see Figure 2). When mult VI VI was shifted to mult VI VT, there was a more rapid and pronounced decrease in the rate of responding during the VT component for both subjects than was observed for the other subjects in Experiment II and Experiment I (see Tables 1 and 2). For SL-1, rate of responding during the VI component was maintained at near baseline levels in Sessions 1-6 and 7-12 and then showed a gradual decrease. SL-2 demonstrated a greater relative decrease in the rate of responding during Sessions 1-6 and 7-12 than did SL-1, but rate during this component recovered to baseline rates during Sessions 13-18.

\section{Discussion}

The results of the separate-reinforcer treatment did not differ consistently from the results of Experiment I. SR-2 demonstrated negative induction during the VI component, but SR-1 did not. Note that all four rats in Experiment I showed the effect.

The separate lever treatment clearly resulted in less interaction between the two components when mult VI VT was introduced following baseline performance on mult VI VI. The only observed interaction between the components was a decrease in rate on the VI component. There is a suggestion of negative induction in the data of SL-1 very late in training. The data of SL-2 were very unstable and indicate an initial induction effect followed by complete recovery.

The cleareast effect observed in the separate-lever treatment was the low rate maintained in the VT component. Rate of responding in the VT component of the mult VI VT decreased to a level equivalent to the asymptotic rate maintained by rats on a simple VT schedule preceded by only $5 \mathrm{~h}$ on its contingent counterpart (Rescorla \& Skucy, 1969). This effect is even more dramatic when considering the longer history of response-dependent reinforcement for SL-1 and SL-2 before shifting to the VT component in the present experiment.

In the separate lever treatment, two factors may be affecting the enhanced independence between the two components of the mult VI VT. They are: (a) the alternating levers may provide more salient discriminative stimuli than the alternating click-on and click-off stimuli correlated with each component in the other treatments and/or (b) different response topographies associated with each lever may provide a decreased tendency for generalization to occur. In general, the results of Experiment II are consistent with the hypothesis that generalization of responding between VI and VT components of a multiple schedule mediate the interactions observed with such schedules.

\section{REFERENCES}

Engberg, L. A. Hansen, G., Welker, R. L., \& Thomas, D. R Acquisition of key-pecking via autoshaping as a function of prior experience: "Learned laziness?" Science, 1972 , 178, $1002-1004$.

Halliday, N. S., \& Boakes, R. A. Behavioral contrast and response independent reinforcement. Journal of the Experimental Analysis of Behavior, 1971, 16, 429-434.

HALliday \& Boakes. 1975.

Huff, R. C., Sherman, J. E., \& Cohn, M. Some effects of response-independent reinforcement on auditory generalization gradients. Journal of the Experimental Analysis of Behavior. 1975, 23, 81-86.

Lattal, K. A., \& Maxey, G. C. Some effects of responseindependent reinforcers on multiple schedules. Journal of the Experimental Analysis of Behavior, 1971, 16, 225-231.

Rescorla, R. A., \& SKUCY, J. C. Effects of responseindependent reinforcers during extinction. Journal of Comparative and Physiological Psychology, 1969, 67, 381-389.

REYNOLDS, G. S. An analysis of interactions in a multiple schedule. Journal of the Experimental Analysis of Behavior, 1961, 4, 107-117.

WEISMAN, R. G., \& RAMSDEN, M. Discrimination of a responseindependent component of a multiple schedule. Journal of the Experimental Analysis of Behavior, 1973, 19, 55-64.

WiLkIE, D. M. Variable time reinforcements in multiple and concurrent schedules. Journal of the Experimental Analysis of Behavior, 1972, 17, 59-66.

(Received for publication August 12, 1975.) 